\title{
Enhancement of Endothelial Function Inhibits Left Atrial Thrombi Development in an Animal Model of Spontaneous Left Atrial Thrombosis
}

\author{
Kazuaki Mawatari, PhD; Emiko Yoshioka, BSc; Satomi Toda, BSc; Sonoko Yasui, PhD; \\ Hiroko Furukawa, PhD; Takaaki Shimohata, PhD; Takamasa Ohnishi, MD, PhD; \\ Masaki Morishima, PhD; Nagakatsu Harada, PhD; Akira Takahashi, MD, PhD; \\ Hiroshi Sakaue, MD, PhD; Yutaka Nakaya, MD, PhD
}

\begin{abstract}
Background: Left atrial (LA) thrombosis is an important cause of systemic embolization. The SPORTS rat model of LA thrombi (Spontaneously-Running Tokushima-Shikoku), which have a unique characteristic of high voluntary wheel running, was previously established. The aim of the present study was to investigate how SPORTS rats develop LA thrombi.
\end{abstract}

\begin{abstract}
Methods and Results: Nitric oxide (NO) produced from cardiovascular endothelial cells plays an important protective role in the local regulation of blood flow, vascular tone, and platelet aggregation. No evidence of atrial fibrillation or hypercoagulability in SPORTS rats regardless of age was found; however, SPORTS rats demonstrated endothelial dysfunction and a decrease of NO production from a young age. In addition, endothelial NO synthase activity was significantly decreased in the LA and thoracic aorta endothelia of SPORTS rats. While voluntary wheel running was able to intermittently increase NO levels, running did not statistically decrease the incidence of LA thrombi at autopsy. However, L-arginine treatment significantly increased NO production and provided protection from the development of LA thrombi in SPORTS rats.
\end{abstract}

Conclusions: They present study results indicate that NO has an important role in the development of LA thrombus, and endothelia pathways could provide new targets of therapy to prevent LA thrombosis. (Circ J 2014; 78: 1980-1988)

Key Words: Atrial fibrillation; Endothelial dysfunction; Nitric oxide; Thrombosis; Vasodilation

$\mathbf{E}$ ndothelial injury/dysfunction, along with hypercoagulability and hemodynamic changes, are characterized as Virchow's triad, and are the major causes that contribute to thrombosis. ${ }^{1,2}$ Left atrial (LA) thrombus formation is an important source of systemic embolization. Cardiogenic embolization, including atrial thrombosis, accounts for more than $15 \%$ of ischemic strokes, ${ }^{3}$ and approximately $30 \%$ of all patients with ischemic stroke or transient ischemic attack are found to have a potential cardiac source of embolism. ${ }^{4}$ Freefloating thrombi, separated from the left atrium, can reach peripheral tissues through blood flow and subsequently induce secondary embolisms, including cerebrovascular events. The development of LA thrombi is frequently found in patients with atrial fibrillation (AF), mitral stenosis, rheumatic heart disease, or long-term complications of the standard orthotopic heart transplantation. ${ }^{5-7}$ Investigation into thrombus formation due to AF has focused on decreased blood flow and the hypercoagulable state in the left atrium. ${ }^{8,9}$ However, although LA stasis and subsequent thrombus formation might be one source of thromboembolism, in cases of AF, this does not seem to provide a complete explanation.

\section{Editorial p 1841}

Endothelial dysfunction has also been reported to play an important role in thrombosis. AF has been shown to cause atrial

Received November 14, 2013; accepted April 16, 2014; released online May 23, 2014 Time for primary review: 17 days

Department of Nutrition and Metabolism (K.M., E.Y., S.T., S.Y., H.F., T.S., N.H., A.T., H.S., Y.N.), Department of Preventive Environment and Nutrition (K.M., T.S., A.T.), Department of Therapeutic Nutrition (S.Y.), Institute of Health Biosciences, the University of Tokushima Graduate School, Tokushima; Department of Nutrition Management, Faculty of Health Science, Hyogo University, Kakogawa (T.O.); and Department of Cardiovascular Sciences, Oita University School of Medicine, Yufu (M.M.), Japan

The first two authors contributed equally to this work (K.M., E.Y.).

Mailing address: Kazuaki Mawatari, PhD, Department of Preventive Environment and Nutrition, Institute of Health Biosciences, the University of Tokushima Graduate School, 3-18-15 Kuromoto-cho, Tokushima 770-8503, Japan. E-mail: mawatari@ nutr.med.tokushima-u.ac.jp

ISSN-1346-9843 doi:10.1253/circj.CJ-13-1398

All rights are reserved to the Japanese Circulation Society. For permissions, please e-mail: cj@j-circ.or.jp 
endocardial dysfunction leading to decreased nitric oxide (NO) levels and reduced endothelial NO synthase (eNOS) expression. ${ }^{10,11} \mathrm{NO}$ is well known as an endothelium-derived relaxation factor, which also plays an important anti-atherosclerotic role. ${ }^{12}$ In addition, NO exerts powerful antithrombotic effects, including inhibition of platelet activation, aggregation, and adhesion to the endothelium. Therefore, reduced NO production might contribute to increased thrombogenicity. ${ }^{12-14}$

From an original Wistar strain, we established the SPORTS (Spontaneously-Running Tokushima-Shikoku) rat model. They have a unique characteristic of high voluntary wheel running, ${ }^{15}$ as well as LA thrombus formation in both male and female SPORTS rats at natural death. ${ }^{16}$ However, this model shows normal sinus rhythm and does not show AF. The aim of the present study was to investigate why LA thrombosis develops in this animal model. We investigated: (1) whether voluntary wheel running changed LA thrombus incidence in SPORTS rats; (2) whether LA thrombi were generated in young SPORTS rats; and (3) whether NO plays an important role in the development of LA thrombi in SPORTS rats. Our results reveal that this model might enable the elucidation of a detailed mechanism for LA thrombus formation and lead to new targets for therapies.

\section{Methods}

\section{Animal Preparation}

We bred male and female SPORTS rats using selective inbreeding in our laboratory, as previously described..$^{15}$ SPORTS rats that ran over 10,000 revolutions on the running wheel per day $(1.07 \mathrm{~m} /$ revolution) were selected for mating. We used male Wistar strain rats as controls (Japan SLC, Shizuoka, Japan). The rats were housed individually in temperature $\left(23 \pm 2^{\circ} \mathrm{C}\right)$ and humidity (50-60\%) controlled rooms, with a dark cycle (20:00-8:00h) and light cycle (8:00-20:00h) daily. We administrated non-purified regular chow (Oriental Yeast, Tokyo, Japan) and tap water ad libitum. We certify that all applicable institutional and governmental regulations concerning the ethical use of animals were followed during this study. This study was conducted in conformity with the guidelines for the care and use of Laboratory animals of the Institute of Health Biosciences, the University of Tokushima Graduate School (No. 08057).

\section{Animal Study 1 - Monitoring of the Incidence of Atrial Thrombi and Life Span in Wheel Running SPORTS Rats}

To investigate the effect of voluntary wheel running on LA thrombus incidence in SPORTS rats, we divided these rats into sedentary or running groups until their natural death (Figure S1A). We compared sedentary SPORTS rats (male, $\mathrm{n}=5$; female, $\mathrm{n}=14$ ), to SPORTS rats housed in cages with a running wheel (male, $n=26$; female, $n=34$ ), until their natural death. The hearts from all animals were immediately removed, cut in half, rinsed to remove residual blood, and checked macroscopically for thrombi formation under a magnifying glass.

\section{Animal Study 2 - Monitoring of Atrial Thrombi Formation and Circulatory, Coagulation, and Metabolic Parameters}

We first compared sedentary male SPORTS vs. the normal Wister rats as a control, which were all housed in normal cages without running wheels ( $n=18$, Figure $\mathbf{S 1 B})$. We examined the histological characteristics and vascular reactivity of the atria and aorta in 20-, 40-, and 60-week-old control and SPORTS rats ( $n=6$ in each group). At 15, 30, 45, and 60 weeks of age, we measured urinary nitrite excretion $(n=6)$. At 20 weeks of age, we measured plasma lipids and performed glucose tolerance tests $(n=6)$, as previously reported. ${ }^{17}$ Prothrombin was assayed at 35 weeks $(n=6)$. At 60 weeks, we monitored electrocardiogram and systolic blood pressure $(n=6)$.

\section{Animal Study 3 - L-Arginine Treatment}

Male 40-week-old normal Wister rats and SPORTS rats, which were housed sedentary in normal cages, were divided into 2 treatment groups, with or without L-arginine treatment ( $n=6$ in each group, Figure S1C). The rats were administered L-arginine dissolved in tap water $(1 \% \mathrm{w} / \mathrm{v})$, ad libitum for 20 weeks.

\section{Histological Analysis}

To observe the development of LA thrombi in SPORTS rats before their death, we harvested tissue from sedentary 20-, 40-, and 60-week-old male SPORTS rats, which were housed in individual normal cages without a running wheel. Rats that were 20,40 , or 60 weeks old were fasted for $16 \mathrm{~h}$ and anesthetized with an intraperitoneal injection of sodium pentobarbital (10 mg/100 g body weight) (Dainippon Sumitomo Pharma, Osaka, Japan). After anesthetization, we immediately collected blood and tissue samples. The hearts were cut in half and rinsed well with saline to remove residual blood. The isolated tissues were fixed in $4 \%$ buffered $p$-formaldehyde for $48 \mathrm{~h}$ and embedded in paraffin, then cut to $3 \mu \mathrm{m}$ in thickness and stained with hematoxylin-eosin or phosphotungstic acid hematoxylin. Images were captured by a CCD camera (DP71; Olympus, Tokyo, Japan) under a light microscope (BX50F4; Olympus). The 2-dimensional areas of thrombus in the LA were measured by using Scion Image software (Scion Image, Frederick, MA, USA).

\section{Urinary Nitrite $\left(\mathrm{NO}_{2}\right)$ Measurement}

Any NO released from endothelial cells is immediately oxidized and then excreted into urine. Therefore, the measurement of $\mathrm{NO}_{2}$ and urinary nitrate $\left(\mathrm{NO}_{3}\right)$ is a convenient assay of NO production. ${ }^{18,19}$ Urine samples were collected at dark periods (20:00-8:00h) and light periods (8:00-20:00 h). Urinary nitrate was converted into $\mathrm{NO}_{2}$ by $\mathrm{NO}_{3}$ reductase. After $\mathrm{NO}_{3}$ reduction, $\mathrm{NO}_{2}$ was measured by using the Griess method commercial kit (Dojindo Laboratories, Kumamoto, Japan).

\section{Vascular Reactivity}

Vascular reactivity of thoracic aortas was measured, as previously described. ${ }^{20}$ Thoracic aortas were removed and placed in cold Krebs-Ringer bicarbonate solution (KRB) containing (in g/L): $\mathrm{NaCl} 6.92, \mathrm{NaHCO}_{3} 2.10, \mathrm{D}$-glucose 2.00, $\mathrm{KH}_{2} \mathrm{PO}_{4}$ $0.16, \mathrm{MgSO}_{4}-7 \mathrm{H}_{2} \mathrm{O} 0.29, \mathrm{KCl} \mathrm{0.35}$, and $\mathrm{CaCl}_{2}$ 0.28. Residual blood and perivascular tissue was removed in cold KRB, and the aortas were cut into ring segments of $3 \mathrm{~mm}$ length. Each ring was then placed in a $3 \mathrm{ml}$ organ bath (Micro Easy Magus; Kishimoto Medical, Kyoto, Japan) and mounted on 2 stainless steel wires, one of which was fastened to the bath and the other connected to a force transducer for the measurement of isometric tension. The bath was filled with $\mathrm{KRB}$ solution at $37^{\circ} \mathrm{C}$ and bubbled with a mixture of $95 \% \mathrm{O}_{2}-5 \% \mathrm{CO}_{2}$. The vasodilative effects by cumulative concentration of acetylcholine (Ach) or sodium nitroprusside (SNP) were measured after vasocontractions, which was induced by $100 \mu \mathrm{mol} / \mathrm{L}$ phenylephrine $(\mathrm{PE})$.

\section{Tissue Preparation and Immunoblotting}

Left atrial and aortic tissues were rapidly isolated from anesthetized rats and residual blood, and adherent tissue was removed in cold phosphate-buffered saline. Tissues were stored at $-80^{\circ} \mathrm{C}$ until the evaluation by immunoblot analyses, as pre- 


\begin{tabular}{|c|c|c|c|c|}
\hline \multirow[b]{3}{*}{ Male SPORTS } & \multirow{2}{*}{$\begin{array}{c}\text { AT } \\
\mathbf{n}(\%)\end{array}$} & \multirow{2}{*}{$\frac{\text { Non-AT }}{\mathrm{n}(\%)}$} & \multicolumn{2}{|c|}{ Total } \\
\hline & & & $\mathbf{n}$ & Lifespan (weeks) \\
\hline & & & & \\
\hline Sedentary & $3(60)$ & $2(40)$ & 5 & $79.7 \pm 14.4$ \\
\hline Running & $14(54)$ & $12(46)$ & 26 & $80.6 \pm 28.2$ \\
\hline \multicolumn{5}{|c|}{ Female SPORTS } \\
\hline Sedentary & $7(50)$ & $7(50)$ & 14 & $117.9 \pm 25.2$ \\
\hline Running & $12(35)$ & $22(65)$ & 34 & $96.6 \pm 27.5$ \\
\hline
\end{tabular}

Male and female SPORTS rats were divided into 2 groups: sedentary groups housed in normal cages, or voluntary running groups housed in cages with a running wheel. Values for the lifespan are shown as mean(weeks) \pm SD. AT, atrial thrombus in the left atrium; SPORTS, Spontaneously-Running Tokushima-Shikoku.

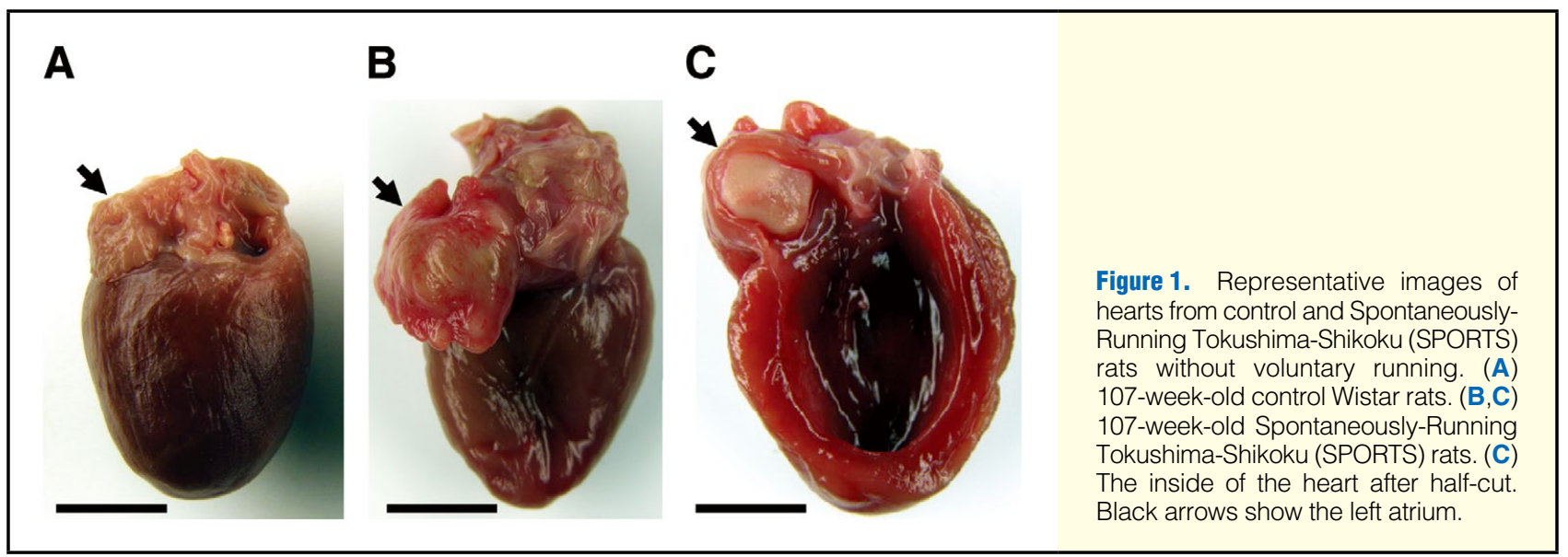

viously descrived. ${ }^{17}$

\section{Reagents}

PE hydrochloride, Ach chloride, L-arginine, and the assay kits for triglycerides (TG), total cholesterol, and high-density lipoprotein (HDL) cholesterol were purchased from Wako Chemicals (Tokyo, Japan). Antibodies against eNOS were purchased from Cell Signaling Technology (Beverly, MA, USA). The antibody against inducible NOS (iNOS) was purchased from BD Transduction Laboratories (Lexington, KY, USA). Antibodies against glyceraldehyde 3-phosphate dehydrogenase and $\alpha$-smooth muscle actin were purchased from Santa Cruz Biotechnology (Santa Cruz, CA, USA).

\section{Statistical Analysis}

Statistical analysis of differences was calculated using ANOVA plus Bonferroni multiple comparison tests. A value of $\mathrm{P}<0.05$ was considered statistically significant. The incidence of LA thrombi, which had 2 categorical variables, was analyzed using the chi-squared test of independence or Fisher's exact probability test.

\section{Results}

\section{Voluntary Running Had Little Effect on the Incidence of LA Thrombi in SPORTS Rats}

The average life spans were not significantly different between the sedentary and running groups (Table 1 ). The 36 to monitored 79 SPORTS rats had some thrombi in the LA (Table 1; Figure 1). In both male and female rats, running groups showed slightly lower rates of LA thrombi than sedentary groups, although the difference was not statistically significant (male rats, $\mathrm{P}=0.60$; female rats, $\mathrm{P}=0.19$ ).

\section{LA Thrombi Were Found in the Early Stage of Life in SPORTS Rats}

At 20 weeks of age, there was no significant difference in the heart weight between control and SPORTS rats; however, the heart weights of SPORTS rats at 40 and 60 weeks were significantly greater than the corresponding age-matched controls (Table S1). In the atria of 20- and 40-week-old rats, a histological examination showed small thrombi in the LA of SPORTS rats but not in control rats (Figure 2). Furthermore, larger-sized thrombi were found in the LA of 60-week-old SPORTS rats (Figures 2H,I). These thrombi consisted of reticulated strands of fibrin and aggregation of red blood cells and platelets (Figures 2C,F,I), which is similar to the histological findings of models of LA thrombosis. ${ }^{21}$ And SPORTS rats had some obvious thickened and injured endothelium in their LA (red arrows in Figures 2F,I). Control rats did not have thrombi in the LA at any age (Figures $\mathbf{2 A , D , G}$ ). These results suggest that LA thrombi are generated starting from early stages of life in SPORTS rats.

\section{SPORTS Rats Did Not Reveal AF or Hypercoagulability}

Cardiac dysrhythmia, especially AF, is well known as one of the major cardiogenic causes for atrial thrombosis. Electrocardiography, monitored by a telemetry transmitter system, did not detect any arrhythmias in SPORTS rats (Figure S2), suggesting that LA thrombi in SPORTS rats were not due to AF. In addition, heart rates were not significantly different be- 
A

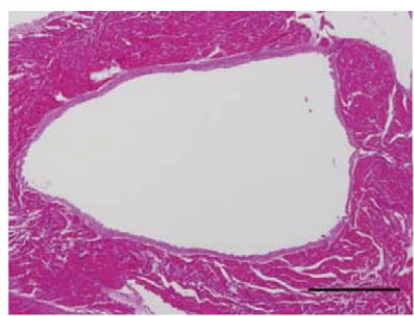

D

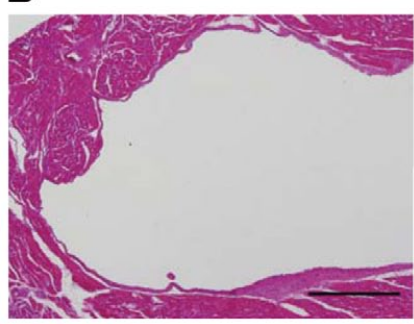

G

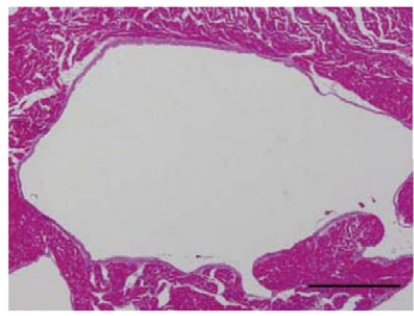

Control (HE)

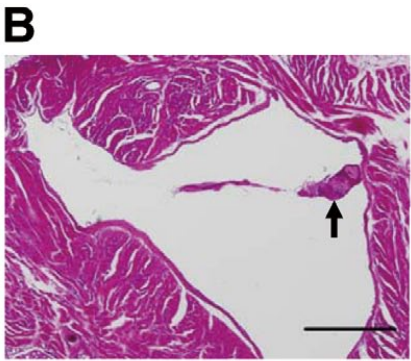

C

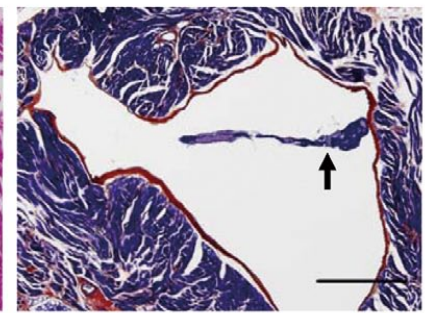

20W

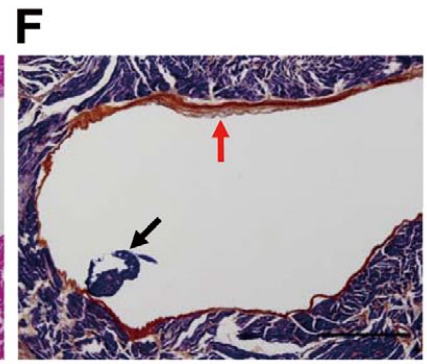

40W

I

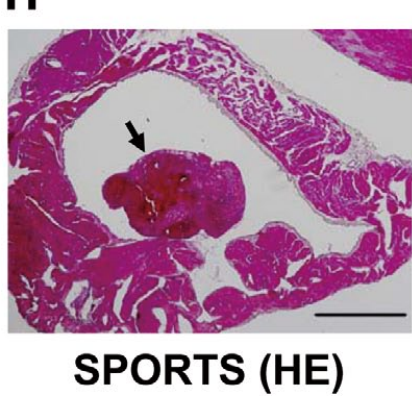

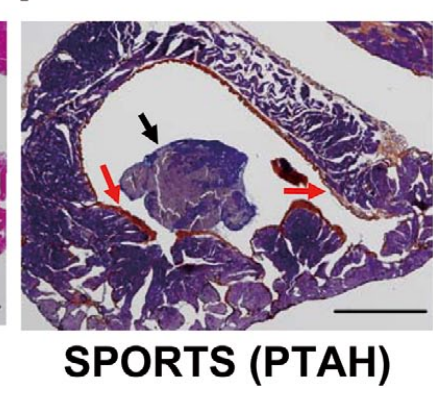

$60 \mathrm{~W}$

Figure 2. Representative microscopic images of the left atria from male control Wistar rats and Spontaneously-Running TokushimaShikoku (SPORTS) rats. (A,D, and G) Control rats. (B,C,E,F,H, and I) SPORTS rats. (A-C) 20-week-old rats. (D-F) 40-week-old rats. (G-I) 60-week-old rats. Sections of the left atria were stained with hematoxylin-eosin (HE) or phosphotungstic acid-hematoxylin (PTAH). Black arrows indicate thrombus formation. Red arrows indicate thickened and injured endothelium. Scale bar $=0.5 \mathrm{~mm}$.

tween control and SPORTS rats (Table 2). Systolic blood pressure in SPORTS rats was only slightly higher than that of control rats (control rats, $107.5 \pm 8.1 \mathrm{mmHg}$; SPORTS rats, $119.9 \pm 12.3 \mathrm{mmHg} ; \mathrm{P}=0.066$ ), indicating that high blood pressure was not a significant cause of LA thrombi (Table 2). In addition, a coagulation assay using prothrombin did not reveal any difference between the 2 groups (Table 2 ).

\section{SPORTS Rats Have Normal Plasma Lipids and Glucose Tolerance}

Because thrombus formation is often associated with lifestylerelated diseases such as hyperlipidemia, hypercholesterolemia, and diabetes mellitus, ${ }^{22}$ we examined plasma lipids and glucose tolerance in SPORTS vs. control rats (Table 2). Although significant differences were not found in the fasting levels of total and HDL cholesterol and insulin, the levels of fasting plasma TG and glucose in SPORTS rats were significantly lower than those of control rats. Cumulative levels (area under the curve) of plasma glucose and insulin during the glucose tolerance test were not different between control and SPORTS rats. These results suggest that development of LA thrombi in SPORTS rats was not due to a disorder of plasma lipids and glucose tolerance.

\section{SPORTS Rats Showed Endothelial Dysfunction in the Thoracic Aorta}

Thrombus formation has been closely associated with endothelium dysfunction. ${ }^{23}$ Vascular endothelial cells have important roles in homeostasis, such as regulation of vascular tone, platelet aggregation, and blood coagulation. ${ }^{14,24}$ Compared to control rats, 20-week-old SPORTS rats showed thickened neointima and thrombus formation in the thoracic aortas (Figures $\mathbf{3 A}, \mathbf{B}$ ). To observe endothelium-dependent vasodilation, we measured the vascular reactivity of isolated thoracic aortas from 20-weekold rats (Figures 3C,D). Compared with controls, SPORTS rats had a significantly decreased Ach-induced vasodilation response (Figure 3C); however, the vasodilative effect of the NO donor, SNP, was not different between control and SPORTS aortas. Therefore, SPORTS rats had decreased endothelium-derived vasodilation. Similarly, endothelium-dependent relaxation was decreased in 40- and 60-week-old SPORTS rats (data not shown). These results suggest that SPORTS rats had endothelium dysfunction in the thoracic aorta. 


\begin{tabular}{|c|c|c|}
\hline & Control & SPORTS \\
\hline \multicolumn{3}{|l|}{ Plasma lipids (20-week-old) } \\
\hline Triglyceride (mg/dl) & $64.2 \pm 5.2$ & $30.7 \pm 3.7^{\star \star \star}$ \\
\hline Total cholesterol (mg/dl) & $65.6 \pm 2.4$ & $65.5 \pm 3.4$ \\
\hline HDL cholesterol (mg/dl) & $31.6 \pm 1.8$ & $33.5 \pm 1.3$ \\
\hline \multicolumn{3}{|l|}{$\begin{array}{l}\text { Glucose tolerance test } \\
\text { (20-week-old) }\end{array}$} \\
\hline Fasting blood glucose (mg/dl) & $70.8 \pm 2.2$ & $58.8 \pm 2.4^{*}$ \\
\hline Glucose AUC $\left(\mathrm{g} \cdot \mathrm{min}^{-1} \cdot \mathrm{ml}^{-1}\right)$ & $14.26 \pm 0.49$ & $13.45 \pm 0.74$ \\
\hline Fasting plasma insulin $(\mathrm{ng} / \mathrm{ml})$ & $0.48 \pm 0.13$ & $0.38 \pm 0.12$ \\
\hline Insulin AUC (ng $\left.\cdot \mathrm{min}^{-1} \cdot \mathrm{ml}^{-1}\right)$ & $218.5 \pm 74.7$ & $201.6 \pm 18.5$ \\
\hline \multicolumn{3}{|l|}{ Coagulation test (35-week-old) } \\
\hline Prothrombin time (s) & $12.0 \pm 0.3$ & $12.4 \pm 0.2$ \\
\hline \multicolumn{3}{|l|}{ BP and heart rate (60-week-old) } \\
\hline Systolic BP (mmHg) & $107.5 \pm 8.1$ & $119.9 \pm 12.3$ \\
\hline Heart rate (beats $/ \mathrm{min}$ ) & $341.4 \pm 10.2$ & $356.5 \pm 16.8$ \\
\hline
\end{tabular}

Male SPORTS and control Wistar rats were sedentarily housed in normal cages. Values are shown as mean \pm SEM $(n=6)$. ${ }^{*} \mathrm{P}<0.05$, significant difference vs. control rats. ${ }^{* \star} \mathrm{P}<0.01$, significant difference vs. control rats. AUC, area under curve; BP, blood pressure; SPORTS, Spontaneously-Running Tokushima-Shikoku; $\mathrm{HDL}$, high-density lipoprotein.

\section{SPORTS Rats Have Downregulated eNOS in the Left Atrium and Thoracic Aorta}

We hypothesized that the development of LA thrombi in SPORTS rats was due to a decrease of NO production, mediated via NO synthase (NOS). Three isoforms of NOS have been identified from independent genes: neuronal (nNOS), inducible (iNOS), and endothelial (eNOS). Several recent reports have demonstrated that eNOS downregulation in the LA was associated with the development of LA thrombi formation. ${ }^{23}$ In both the LA and aortas, SPORTS rat eNOS expression was significantly decreased compared to tissues from control rats, although there was no difference in iNOS expression between control and SPORTS rats (Figure 4). Furthermore, urinary $\mathrm{NO}_{2}$ excretion from sedentary SPORTS rats was significantly lower than that of control rats, consistent from 15 to 60 weeks of age (Figure 5). From these results, we conclude that the decrease of NO production in SPORTS rats is due to the downregulation of eNOS expression, possibly explaining the development of LA thrombi in the SPORTS model.

\section{L-Arginine Treatment Increased NO Production and Decreased the Development of LA Thrombi}

$\mathrm{NO}$ is synthesized from L-arginine as an enzymatic substrate of NOS. L-arginine treatment increases NO production and has protective effects for the local regulation of blood flow, vascular tension, and platelet aggregation of endothelial cells. ${ }^{25}$ To observe the effect of L-arginine treatment on NO produc-
A

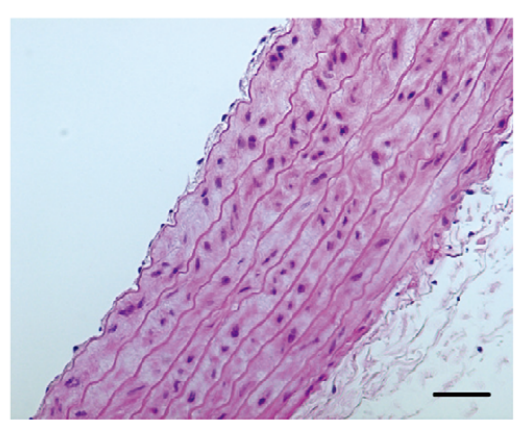

C

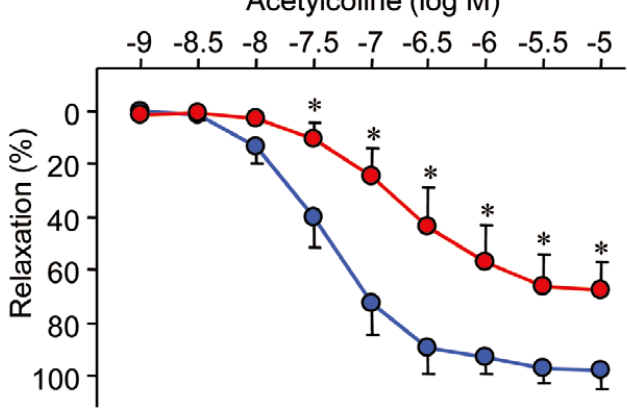

B

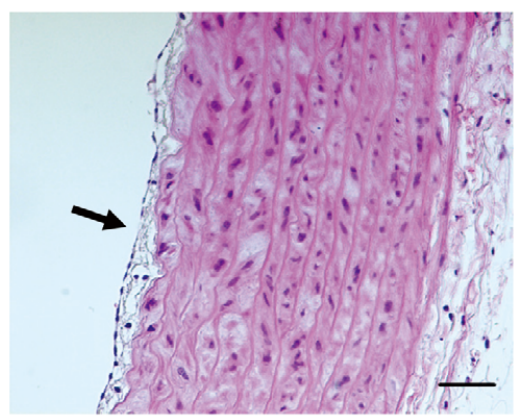

D

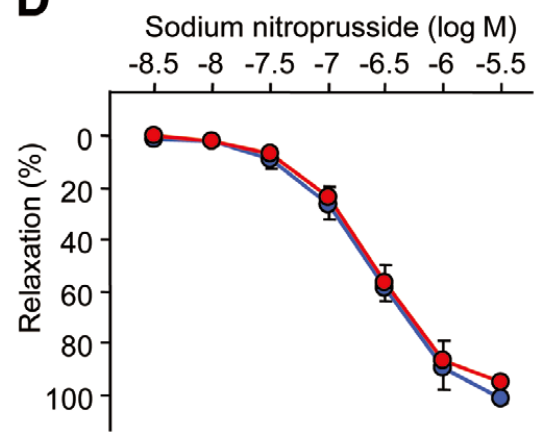

Figure 3. Morphological changes and vascular reactivity of thoracic aortas in 20-week-old control Wistar rats and Spontaneously-Running Tokushima-Shikoku (SPORTS) rats. Representative microscopic images of thoracic aortas from (A) Control rat or (B) SPORTS rat. The aortic sections were stained with hematoxylin-eosin. Scale bar $=50 \mu \mathrm{m}$. Vascular reactivity of thoracic aortas with (C) acetylcholine (Ach)-induced vasodilation. (D) Sodium nitroprusside (SNP)-induced vasodilation in both control rats (Blue) and SPORTS rats (Red). The aortic tension was measured in an organ chamber. Aortas were pre-contracted with $100 \mu \mathrm{mol} / \mathrm{L}$ phenyrephrine, then treated with Ach or SNP at indicated concentrations. Values are shown as mean \pm SEM $(n=6)$. ${ }^{*}$ Difference $(P<0.05)$ vs. control rats. 
A
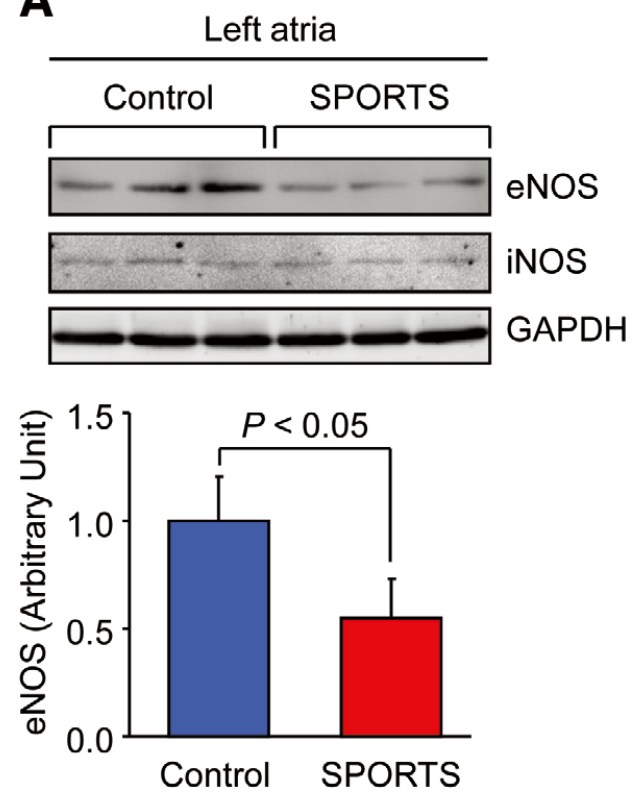

B
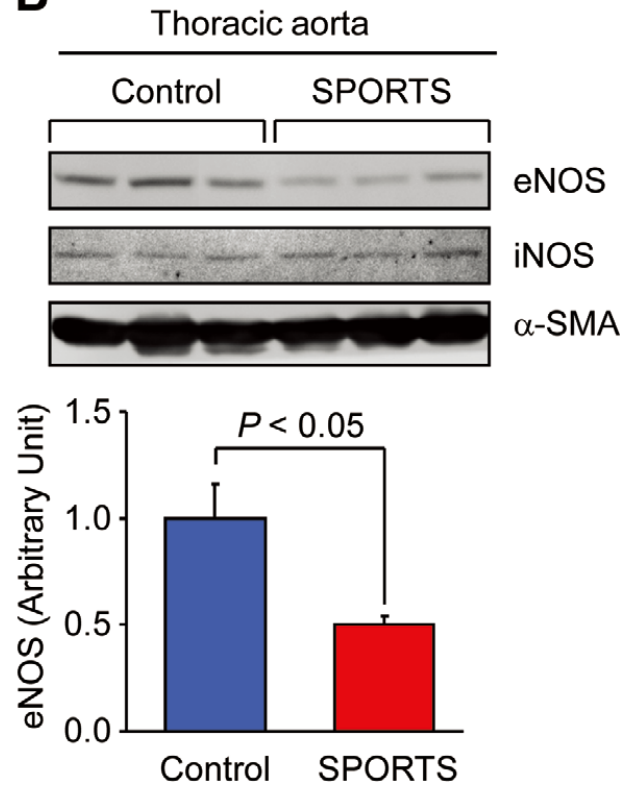

Figure 4. Expression of nitric oxide synthase (NOS) in the left atrium and aortic tissues isolated from 20-week-old control Wistar rats and Spontaneously-Running Tokushima-Shikoku (SPORTS) rats. (A) Left atria. (B) Thoracic aortas. Endothelial NOS (eNOS) and inducible NOS (iNOS) protein levels were determined by Western blotting. Glyceraldehyde 3-phosphate dehydrogenase (GAPDH) or a-smooth muscle actin (SMA) was used as loading controls. The data of eNOS expression were analyzed by densitometric quantification. Values are shown as mean $\pm \operatorname{SEM}(n=6)$.

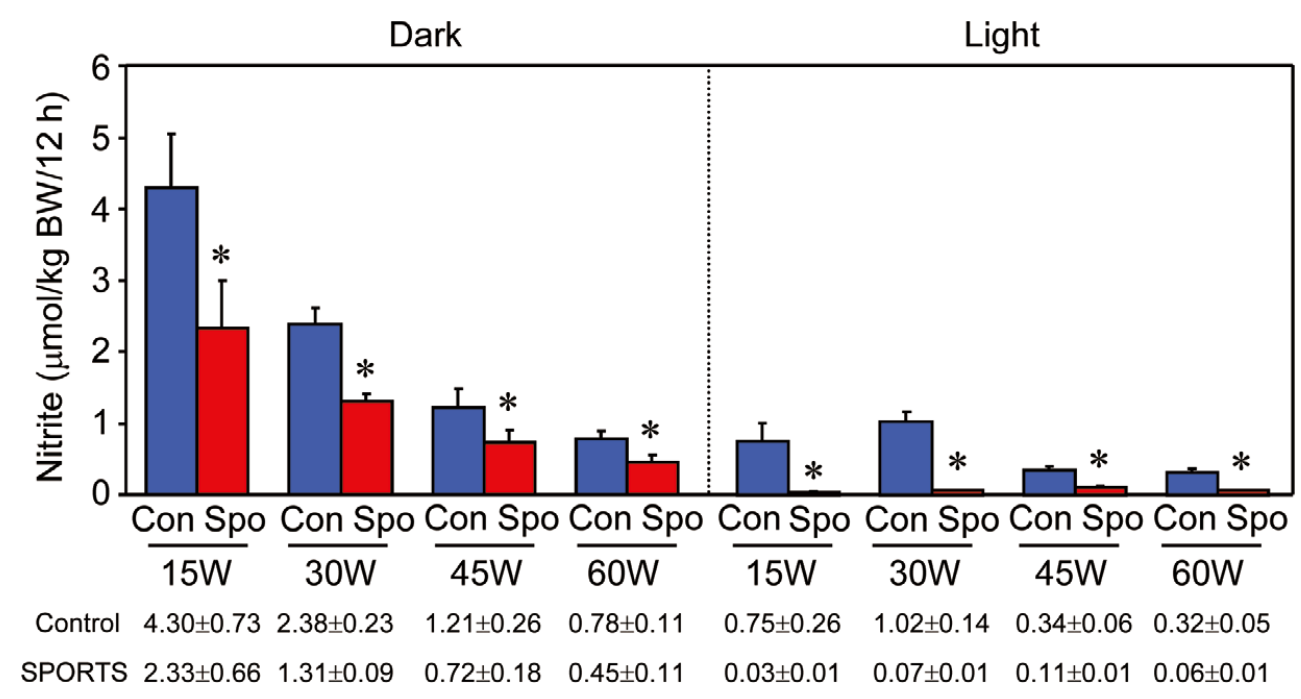

Figure 5. Urinary nitrite $\left(\mathrm{NO}_{2}\right)$ excretion levels in control Wistar rats and Spontaneously-Running Tokushima-Shikoku (SPORTS) rats. (Blue bars) Control rats. (Red bars) SPORTS rats. Urine samples were collected at both dark periods (20:00-8:00 h) and light periods (8:00-20:00 h) in the indicated weeks $(W)$. Values are shown as mean $\pm S E M(n=6)$. *Significant difference $(P<0.05)$ vs. control rats.

tion, we measured urinary $\mathrm{NO}_{2}$ excretion. Treatment with Larginine significantly increased urinary $\mathrm{NO}_{2}$ in SPORTS rats during both dark and light periods (Figure 6A). Next, we investigated the effect of L-arginine treatment on the development of LA thrombi. The 2-dimensional area of the LA throm- bi section in SPORTS rats was significantly decreased by L-arginine treatment (Figure 6B). From these results, we suggest that the development of LA thrombi is due to a decrease of NO production, and that L-arginine treatment provides protection against LA thrombosis. 
A

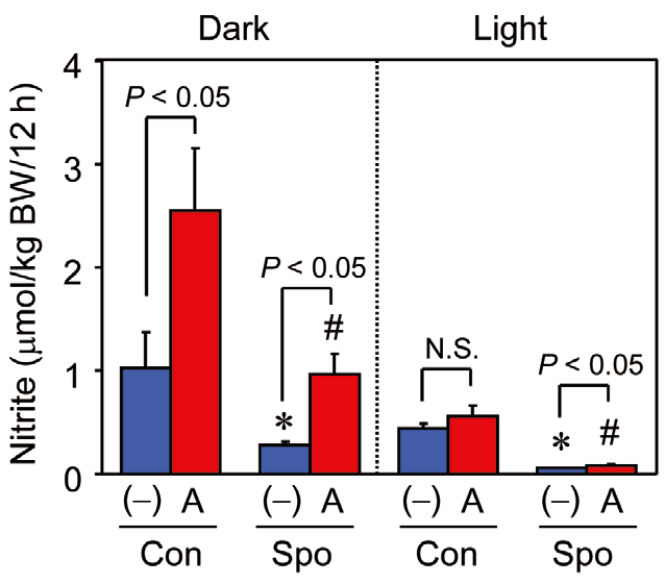

$\begin{array}{llllll}(-) & 1.03 \pm 0.35 & 0.27 \pm 0.04 & 0.39 \pm 0.05 & 0.05 \pm 0.01\end{array}$

$\begin{array}{lllll}\text { L-arginine } \quad 2.54 \pm 0.61 & 0.96 \pm 0.20 & 0.55 \pm 0.11 & 0.08 \pm 0.01\end{array}$

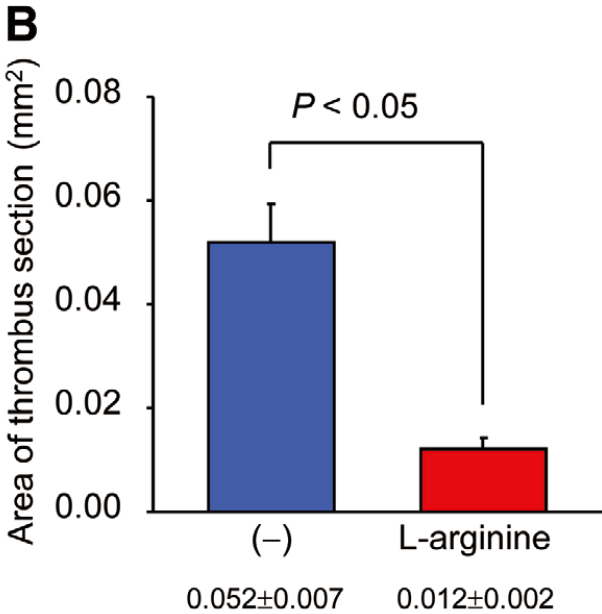

Figure 6. Effect of L-arginine treatment for 20 weeks in 60-week-old control Wistar rats and Spontaneously-Running TokushimaShikoku (SPORTS) rats. (A) Urinary nitrite excretion levels. (B) Development of left atrial thrombus. (Blue bars) Non-treated control groups. (Red bars) L-arginine supplemented groups. Values are shown as mean $\pm S E M(n=6$ in each group). *Significant difference $(P<0.05)$ vs. control rats. "Significant difference $(P<0.05)$ vs. L-arginine treated control rats.

\section{Discussion}

Cardiac dysrhythmia, especially AF, is a well-known cause of LA thrombus formation. In the present study, we found LA thrombi in young as well as adult SPORTS rats, without AF or hypercoagulability. SPORTS rats had endothelial dysfunction and decreased NO production from a young age. In addition, eNOS expression was significantly decreased in the LA and thoracic aortas of SPORTS rats. NO produced from endothelial cells plays important protective roles in the local regulation of blood flow, vascular tone, and platelet aggregation. ${ }^{14,24}$ From these data, we suggest that the development of LA thrombi might, at least in part, be due to decreased NO production. Furthermore, we found that L-arginine treatment significantly increased NO production and had protective effects for the development of LA thrombus in SPORTS rats. Recent reports found that LA thrombi formation induced by AF might be associated with a marked decrease in eNOS expression and NO bioavailability in the LA. ${ }^{26,27}$ Our results indicate that $\mathrm{NO}$ has an important role in the development of LA thrombus, regardless of the fibrillation state of the atria.

In this study, we had just focused on relatively old thrombus in the left atrium. Old thrombi, which have a rigid structure, are highly organized, mostly by collagen in the perpetuation stage. In contrast, fresh thrombi, which are still in the development and expansion stage, consist mainly of blood material and are not yet organized by collagen. ${ }^{28}$ These fresh thrombi are more elastic and have a lower stiffness, therefore, we immediately cut the hearts in half and rinsed them well with saline to removing residual blood before fixation. In the vasculature, endothelial dysfunction is also known to play an important role in thrombus development, including by decreasing blood flow and hypercoagulability, as shown in the wellknown Virchow's triad. ${ }^{29}$ Recent experimental and clinical studies have demonstrated that Virchow's triad is also important in thrombus formation in the fibrillating left atrium..$^{23,26,27,30}$
Obvious thickened and injured endothelium of the LA surface in SPORTS rats was observed by histological analyses (Figures 2F,I). von Willebrand factor (vWf), a well-established index of endothelial damage and dysfunction, associate to the platelets via the matrix formation with glycoprotein Ib/IX. ${ }^{31}$ vWf was significantly increased in the injured atrial endothelium containing inflammatory cells in patients with AF who have a history of cardiogenic thromboembolism. ${ }^{32}$ From these reports, vWf might be a key factor of LA thrombosis in SPORTS rats. Several recent reports have demonstrated that $\mathrm{AF}$, per se, causes atrial endocardial dysfunction, which is manifested by increased plasminogen activator inhibitor-1 expression, decreased expression of eNOS, tissue factor pathway inhibitor, and thrombomodulin in the LA endocardium, leading to local coagulation imbalance on the internal surface of the atrium. ${ }^{23,27}$ Another report reported that inflammation might not only result in endothelial damage and dysfunction but might also be linked directly to thrombogenesis. ${ }^{33}$ Our previous study indicated that many neutrophils accumulated around each thrombus, and some neutrophils and lymphocytes were observed to infiltrate the atrial wall in SPORTS rats. ${ }^{16}$ Interleukin 6, a cytokine produced from these inflammatory cells, increases sensitivity to thrombin and stimulates transcription of fibrinogen. ${ }^{34,35}$ These reports suggest that the imbalance of coagulation on the internal surface of the atrium is an important factor for atrial thrombus formation.

However, some questions about this animal model remain unclear in this study. There were no thrombi in right atrium (RA) of natural dead SPORTS rats in this study (data not shown). A clear reason why thrombus formation was limited in the LA remains unknown. In healthy young subjects with normal rhythm, blood pressure, and flow, the LA appears to avoid any sustained stasis of blood. ${ }^{36}$ Atrial thrombosis was related with some factors such as stasis of blood flow and hypercoagulable status. ${ }^{8} \mathrm{AF}$ is closely associated with these factors and an increased risk of both LA and RA thrombosis, ${ }^{5,37}$ but the number 
of reports about thrombosis in the LA is higher than that for RA. Furthermore, in an AF model and patients, endothelial dysfunction, containing a decrease in NO production or a increase in VWF expression, was observed only in the LA and not in the RA. ${ }^{26,38}$ From these reports, LA might be easy to suffer endothelial dysfunction and thrombosis rather than RA. Formation of LA thrombi is known to be an important risk factor for cerebral or the other embolism. ${ }^{39}$ In the progress study, our collaborators observed paralytic symptoms in some SPORTS rats, but a question that depends on thromboembolism in the brain or not remains unclear. In addition, one of main causes of death in SPORTS rats seemed to be heart failure, but the actual numbers of each cause of death were not monitored in this study. Our collaborators observed that both atrial natriuretic peptide and brain natriuretic peptide were increased in the heart tissue of SPORTS rat (unpublished data), but it remains unclear why SPORTS rats cause heart failure. We thought that two of the factors was a slightly higher blood pressure and heart rate than control rats (Table 2), which might be caused by a high catecholamine condition. ${ }^{17,40}$ More detailed analyses of the heart, brain, and other tissues in SPORTS rats are necessary in the progress study.

In this study, we established a new model of LA thrombosis with decreased NO production, but without AF. Adrie et al reported in an animal study that inhaled NO might inhibit the development of coronary thrombosis. ${ }^{41}$ Another report found that plasma NO levels were significantly increased after exercise compared with that of sedentary rats. ${ }^{42}$ In addition, there is increasing evidence that regular exercise reduces the risk of arterial and venous thrombotic events, ${ }^{43}$ due to reduced circulating levels of viscosity factors and inflammatory variables. ${ }^{44,45}$ In our model, voluntary wheel running increased NO levels intermittently (data not shown), but could not significantly decrease the incidence of LA thrombi. From these results, the type of exercise to constantly increase NO levels might be an important factor in preventing atrial thrombosis. Subsequently, we found that L-arginine treatment significantly increased NO production and efficiently inhibited the development of LA thrombus. Our data were supported by other reports that found the supplement of L-arginine to ameliorate the endothelial dysfunction and thrombus formation in models of both arterial and venous thrombosis. ${ }^{46,47}$ Hydroxymethylglutaryl (HMG)CoA reductase inhibitors and omega- 3 fatty acids, which are known to improve endothelial dysfunction, show the protective effect in animal models of $\mathrm{AF},{ }^{48}$ but the results, which these have the effectiveness or not, remain controversial in $\mathrm{AF}$ patients. ${ }^{49,50}$ Therefore, it is still unknown which therapies focused on endothelial dysfunction alone, and which therapies were used that were similar to this study that could recover LA thrombosis completely in AF patients. Shin et al reported that catheter ablation for AF patients with no recurrence improved endothelial dysfunction by the measurement of flow-mediated dilation (FMD), but they needed for 6 months to the FMD score of control subject. ${ }^{51}$ Furthermore, the patients with recurrence continue endothelial dysfunction after AF ablation. ${ }^{51}$ However, some reports showed that HMG-CoA reductase inhibitors and omega- 3 fatty acids had protective effectives for AF patients post-cardioversion or ablation. ${ }^{52,53}$ We thought that the animal model without AF we used in this study is similar to that of patients post-cardioversion or ablation, and some continuous anticoagulant therapies or treatment for endothelial dysfunction might be beneficial in these patients without an AF condition. The results of the present study provide details towards deciphering a new model of LA thrombosis, as well as a new direction towards targets of therapy to prevent
LA thrombosis.

\section{Acknowledgments}

This study was supported, in part, by the Grant-in-Aid for Scientific Research to Y. Nakaya (No. 19300222) and to K. Mawatari (No. 24500857) from the Ministry of Education, Science, Sports, and Culture and Technology, Japan.

\section{References}

1. Virchow R. Phlogose und thrombose im Gefäßsystem. Gesammelte Abhandlungen zur Wissenschaftlichen Medizin. Frankfurt: Staatsdruckerei, 1856.

2. Armstrong W, Golan DE, Tashjian AH, Armstrong E. Principles of pharmacology: The pathophysiologic basis of drug therapy. Philadelphia: Wolters Kluwer Health/Lippincott Williams \& Wilkins, 2008; 396.

3. Vinga C, de Rito V, Criconia GM, Russo A, Testa M, Fanell R, et al. Left atrial thrombus and spontaneous echo contrast in nonaniticoagulated mitral stenosis: A transesophageal echocardiographic study. Chest 1993; 103: 348-352.

4. Cardiogenic brain embolism: The second report of the Cerebral Embolism Task Force. Arch Neurol 1989; 46: 727-743.

5. Pisters R, Lane DA, Marin F, Camm AJ, Lip GY. Stroke and thromboembolism in atrial fibrillation. Circ J 2012; 76: 2289-2304.

6. Ozer N, Tokgözoğlu L, Ovünç K, Kabakçi G, Aksöyek S, Aytemir $\mathrm{K}$, et al. Left atrial appendage function in patients with cardioembolic stroke in sinus rhythm and atrial fibrillation. J Am Soc Echocardiogr 2000; 13: 661-665.

7. Dubrava J, Garay R. The role of transesophageal echocardiography in detection of cardiogenic and aortic sources of embolism in stroke and transient ischaemic attacks. Vnitr Lek 2006; 52: 144-151.

8. Fatkin D, Kelly RP, Feneley MP. Relations between left atrial appendage blood flow velocity, spontaneous echo cardiographic contrast and thromboembolic risk in vivo. J Am Coll Cardiol 1994; 23: 961-969.

9. Gustafsson C, Blomback M, Britton M, Hamsten A, Svensson J. Coagulation factors and the increased risk of stroke in nonvalvular atrial fibrillation. Stroke 1990; 21: 47-51.

10. Yamashita T, Sekiguchi A, Kato T, Tsuneda T, Iwasaki Y, Sagara $\mathrm{K}$, et al. Angiotensin type 1 receptor blockade prevents endocardial dysfunction of rapid paced atria in rats. J Renin Angiotensin Aldosterone Syst 2007; 8: 127-132.

11. Joseph G, Walter J, David JW. Acute onset human atrial fibrillation is associated with local cardiac platelet activation and endothelial dysfunction. J Am Coll Cardiol 2007; 51: 1790-1793.

12. Moncada S, Palmer RM, Higgs EA. Nitric oxide: Physiology, pathophysiology, and pharmacology. Pharmacol Rev 1991; 43: 109-142.

13. Lefer AM, Lefer DJ. The role of nitric oxide and cell adhesion molecules on the microcirculation in ischaemia-reperfusion. Cardiovasc Res 1996; 32: 743-751.

14. Alonso D, Ranomski MW. Nitric oxide, platelet function, myocardial infarction and reperfusion therapies. Heart Fail Rev 2008; 8: 47-54.

15. Morishima-Yamato M, Hisaoka F, Shinomiya S, Harada N, Matoba $\mathrm{H}$, Takahashi A, et al. Cloning and establishment of a line of rats for high levels of voluntary wheel running. Life Sci 2005; 77: 551-561.

16. Ohnishi T, Hisaoka F, Morishima M, Takahashi A, Harada N, Mawatari K, et al. Establishment of a model of Spontaneously-RunningTokushima-Shikoku rats with left atrial thrombosis. J Toxicol Pathol 2014; 27: 51-56.

17. Hattori A, Mawatari K, Tsuzuki S, Yoshioka E, Toda S, Yoshida M, et al. Beta-adrenergic-AMPK pathway phosphorylates acetyl-CoA carboxylase in a high-epinephrine rat model, SPORTS. Obesity 2010; 18: $48-54$.

18. Calo L, Cantaro S, Paleari D, Vianello D, Zerbo F, Bonfante L, et al. Urinary $\mathrm{NO}_{2}{ }^{-}$and $\mathrm{NO}_{3}{ }^{-}$evaluation by an ion chromatography system. Biomed Chromatogr 1998; 12: 97-98.

19. Suzuki S, Ikenaga H, Hishikawa K, Nakaki T, Kato R, Saruta T. Increases in $\mathrm{NO}_{2}^{-} / \mathrm{NO}_{3}{ }^{-}$excretion in the urine as an indicator of the release of endothelium-derived relaxing factor during elevation of blood pressure. Clin Sci (Lond) 1992; 82: 631-634.

20. Mawatari K, Kakui S, Harada N, Ohnishi T, Niwa Y, Okada K, et al. Endothelin-1(1-31) levels are increased in atherosclerotic lesions of the thoracic aorta of hypercholesterolemic hamsters. Atherosclerosis 2004; 175: 203-212.

21. Anderson JR. Disturbances of blood flow and body fluids. In: Anderson JR, editor. Muir's Textbook of Pathology, 11th edn. London, UK: Edward Arnold, 1980; 226-268.

22. Alessi MC, Juhan-Vague I. Metabolic syndrome, haemostasis and 
thrombosis. Thromb Haemost 2008; 99: 995-1000.

23. Fukuchi M, Watanabe J, Kumagai K, Katori Y, Baba S, Fukuda K, et al. Increased von Willebrand factor in the endocardium as a local predisposing factor for thrombogenesis in overloaded human atrial appendage. J Am Coll Cardiol 2001; 37: 1436-1442.

24. Sharmalar R, Chirkov YY. Platelet hyperaggregability: Impaired responsiveness to nitric oxide ("Platelet NO Resistance") as a therapeutic target. Cardiovasc Drugs Ther 2008; 22: 193-203.

25. Xiao XM, Li LP. L-Arginine treatment for asymmetric fetal growth restriction. Int J Gynaecol Obstet 2005; 88: 15-18.

26. Cai H, Li Z, Goette A, Mera F, Honeycutt C, Feterik K, et al. Downregulation of endocardial nitric oxide synthase expression and nitric oxide production in atrial fibrillation: Potential mechanisms for atrial thrombosis and stroke. Circulation 2002; 106: 2854-2858.

27. Yamashita T, Sekiguchi A, Iwasaki YK, Sagara K, Hatano S, Iinuma $\mathrm{H}$, et al. Thrombomodulin and tissue factor pathway inhibitor in endocardium of rapidly paced rat atria. Circulation 2003; 108: 24502452.

28. Brass LF, Stalker TJ, Zhu L, Woulfe DS. Signal transduction during platelet plug formation. In: Michelson A, editor. Platelets, 2nd edn. San Diego: Academic Press, 2007; 319-346.

29. Chung I, Lip GY. Vichow's triad revisited: Blood constituents. Pathophysiol Haemost Thromb 2003; 33: 449-454.

30. Shirani J, Alaeddini J. Structural remodeling of the left atrial appendage in patients with chronic non-valvular atrial fibrillation: Implications for thrombus formation, systemic embolism and assessment by transesophageal echocardiography. Cardiovasc Pathol 2000; 9: 95-101.

31. Bergmeier W, Chauhan AK, Wagner DD. Glycoprotein Ibalpha and von Willebrand factor in primary platelet adhesion and thrombus formation: Lessons from mutant mice. Thromb Haemost 2008; 99: $264-270$.

32. Nakamura Y, Nakamura K, Fukushima-Kusano K, Ohta K, Matsubara $\mathrm{H}$, Hamuro T, et al. Tissue factor expression in atrial endothelia associated with nonvalvular atrial fibrillation: Possible involvement in intracardiac thrombogenesis. Thromb Res 2003; 111: 137-142.

33. Boos CJ, Anderson RA, Lip GY. Is atrial fibrillation an inflammatory disorder? Eur Heart J 2006; 27: 136-149.

34. Burstein SA. Cytokines, platelet production and hemostasis. Platelets 1997; 8: $93-104$

35. Amrani DL. Regulation of fibrinogen biosynthesis: Glucocorticoid and interleukin-6 control. Blood Coagul Fibrinolysis 1990; 1: 443446.

36. Fluckiger JU, Goldberger JJ, Lee DC, Ng J, Lee R, Goyal A, et al. Left atrial flow velocity distribution and flow coherence using fourdimensional FLOW MRI: A pilot study investigating the impact of age and Pre- and Postintervention atrial fibrillation on atrial hemodynamics. J Magn Reson Imaging 2013; 38: 580-587.

37. Kronik G; the European Working Group on Echocardiography. The European Cooperative Study on the clinical significance of right heart thrombi: European Working Group on Echocardiography. Eur Heart J 1989; 10: 1046-1059.

38. Fukuchi M, Watanabe J, Kumagai K, Katori Y, Baba S, Fukuda K, et al. Increased von Willebrand factor in the endocardium as a local predisposing factor for thrombogenesis in overloaded human atrial appendage. J Am Coll Cardiol 2001; 37: 1436-1442.

39. Inoue $\mathrm{H}$, Okumura $\mathrm{K}$, Atarashi $\mathrm{H}$, Yamashita $\mathrm{T}$, Origasa $\mathrm{H}$, Kumagai $\mathrm{N}$, et al. Target international normalized ratio values for preventing thromboembolic and hemorrhagic events in Japanese patients with non-valvular atrial fibrillation: Results of the J-RHYTHM Registry. Circ J 2013; 77: 2264-2270.
40. Morishima M, Harada N, Hara S, Sano A, Seno H, Takahashi A, et al. Monoamine oxidase A activity and norepinephrine level in hippocampus determine hyperwheel running in SPORTS rats. Neuropsychopharmacology 2006; 31: 2627-2638.

41. Adrie C, Bloch KD, Moreno PR, Hurford WE, Guerrero JL, Holt R, et al. Inhaled nitric oxide increases coronary artery patency after thrombolysis. Circulation 1996; 94: 1919-1926.

42. Sun MW, Zhong MF, Gu J, Qian FL, Gu JZ, Chen H. Effect of different levels of exercise volume on endothelium-dependent vasodilation: Roles of nitric oxide synthase and heme oxygenase. Hypertension Res 2008; 31: 805-816.

43. Lowe GD. Virchow's triad revisited: Abnormal flow. Pathophysiol Haemost Thromb 2004; 33: 455-457.

44. Leng GC, Fowler B, Ernst E. Exercise for intermittent claudication. Cochrane Database Syst Rev 2008; 8: CD000990, doi:10.1002/14651858.CD000990.pub2.

45. Wang JS. Exercise prescription and thrombogenesis. J Biomed Sci 2006; 13: 753-761.

46. Davis MR, Ortegon DP, Kerby DJ, Ignarro LJ, Kashyap VS. Endothelial dysfunction after arterial thrombosis is ameliorated by L-arginine in combination with thrombolysis. J Vasc Interv Radiol 2003; 14: $233-239$.

47. Lin PH, Johnson CK, Pullium JK, Bush RL, Conklin BS, Chen C, et al. L-arginine improves endothelial vasoreactivity and reduces thrombogenicity after thrombolysis in experimental deep venous thrombosis. J Vasc Surg 2003; 38: 1396-1403.

48. Takahashi N, Kume O, Wakisaka O, Fukunaga N, Teshima Y, Hara $\mathrm{M}$, et al. Novel strategy to prevent atrial fibrosis and fibrillation. Circ J 2012; 76: 2318-2326.

49. Zhou X, Du JL, Yuan J, Chen YQ. Statin therapy is beneficial for the prevention of atrial fibrillation in patients with coronary artery disease: A meta-analysis. Eur J Pharmacol 2013; 707: 104-111.

50. Mariani J, Doval HC, Nul D, Varini S, Grancelli H, Ferrante D, et al. $\mathrm{N}-3$ polyunsaturated fatty acids to prevent atrial fibrillation: Updated systematic review and meta-analysis of randomized controlled trials. J Am Heart Assoc 2013; 2: e005033, doi:10.1161/JAHA.112.005033.

51. Shin SY, Na JO, Lim HE, Choi CU, Choi JI, Kin SH, et al. Improved endothelial function in patients with atrial fibrillation through maintenance of sinus rhythm by successful catheter ablation. $J$ Cardiovasc Electrophysiol 2011; 22: 376-382.

52. Costanzo S, di Niro V, Di Castelnuovo A, Gianfagna F, Donati MB, de Gaetano G, et al. Prevention of postoperative atrial fibrillation in open heart surgery patients by preoperative supplementation of $n-3$ polyunsaturated fatty acids: An updated meta-analysis. $J$ Thorac Cardiovasc Surg 2013; 146: 906-911.

53. Naji F, Sabovic M. The current role of statin therapy in the treatment of atrial fibrillation. Cardiovasc Hematol Agents Med Chem 2013; 11: $9-13$.

\section{Supplementary Files}

Supplementary File 1

Figure S1. Schema of this study using an animal model.

Figure S2. Representative averaged electrocardiography (ECG) traces in 60-week-old (A) control Wistar rats and (B) SpontaneouslyRunning Tokushima-Shikoku (SPORTS) rats.

Table S1. Body and tissue weights of control and SPORTS rats

Please find supplementary file(s);

http://dx.doi.org/10.1253/circj.CJ-13-1398 